\title{
Antipneumococcal Activity of LBM415, a New Peptide Diformylase Inhibitor, Compared with Those of Other Agents
}

\author{
Lois M. Ednie, Glenn Pankuch, and Peter C. Appelbaum* \\ Department of Pathology, Hershey Medical Center, Hershey, Pennsylvania
}

Received 4 June 2004/Returned for modification 16 June 2004/Accepted 24 June 2004

\begin{abstract}
The MICs of LBM415, a new peptide diformylase inhibitor, were evaluated and ranged from 0.03 to 4.0 $\mu \mathrm{g} / \mathrm{ml}$ for 300 pneumococci, irrespective of their $\beta$-lactam, macrolide, and quinolone susceptibilities. By comparison, vancomycin, teicoplanin, linezolid, and quinupristin-dalfopristin were also active, with MICs $\leq 2.0$ $\mu \mathrm{g} / \mathrm{ml}$. Gatifloxacin and moxifloxacin were the most active quinolones tested, while the MICs of the $\beta$-lactams rose with those of penicillin G. LBM415 at two times the MIC was bactericidal $(99.9 \%$ killing) against six strains after $24 \mathrm{~h}$.
\end{abstract}

The incidence of pneumococci resistant to penicillin $\mathrm{G}$ and other $\beta$-lactams and non- $\beta$-lactams has increased worldwide, including the United States, at an alarming rate $(3,6,10,11)$. The higher the penicillin G MIC is, the more likely it is that pneumococci will also be resistant to macrolides and other unrelated agents (11). The recent emergence of quinoloneresistant pneumococci (15) has further complicated the therapeutic problem. There is an urgent need for oral compounds for the outpatient treatment of respiratory tract infections caused by resistant pneumococci (6).

LBM415 (previously known as NVP-PDF713) is a new peptide deformylase inhibitor with excellent activities against gram-positive bacteria $(1,2,7,12,13)$. The present study examined (i) the antipneumococcal activities of LBM415 compared with those of penicillin $\mathrm{G}$, amoxicillin, amoxicillin-clavulanate, imipenem, meropenem, ceftriaxone, cefuroxime, cefpodoxime, cefdinir, ciprofloxacin, levofloxacin, gatifloxacin, moxifloxacin, azithromycin, clarithromycin, linezolid, quinupristin-dalfopristin, vancomycin, and teicoplanin against 300 pneumococci by the agar dilution MIC methodology and (ii) the activities of the compounds listed above plus daptomycin against 12 pneumococci by time-kill analysis.

The pneumococci used for the agar dilution studies comprised 80 penicillin-susceptible, 88 penicillin-intermediate, and 132 penicillin-resistant strains. Of these, 154 were macrolide resistant and had the following mechanisms of resistance: $\operatorname{erm}(\mathrm{B}), 87$ isolates; $\operatorname{mef}(\mathrm{A}), 40$ isolates; $\operatorname{erm}(\mathrm{B})$ and $m e f(\mathrm{~A}), 1$ isolate; L4 protein mutation, 18 isolates; 23S rRNA mutation, 4 isolates; and erm(A), 4 isolates. Forty-two penicillin-susceptible but macrolide-resistant strains were chosen for this study. Thirty strains were quinolone resistant (levofloxacin MICs, $\geq 4$ $\mu \mathrm{g} / \mathrm{ml}$ ) and had defined mutations in the type II topoisomerase enzymes. Twelve pneumococcal strains were tested in the timekill studies: four penicillin-sensitive strains, two intermediate strains, and six penicillin-resistant strains. Of these 12 strains, 10 were macrolide resistant [4 had $\operatorname{erm}(\mathrm{B}), 4$ had $\operatorname{mef}(\mathrm{A})$, and

\footnotetext{
* Corresponding author. Mailing address: Department of Pathology, Penn State Hershey Medical Center, 500 University Dr., H160, Hershey, PA 17033. Phone: (717) 531-5113. Fax: (717) 531-7953. E-mail: pappelbaum@psu.edu.
}

2 had an L4 protein mutation] and 2 were quinolone resistant. LBM415 powder for susceptibility testing was obtained from Novartis Research Laboratories, Hanover, N.J. The other antimicrobials were obtained from their respective manufacturers. The agar dilution method was performed with MuellerHinton agar (BBL Microbiology Systems, Cockeysville, Md.) supplemented with 5\% sheep blood. Although NCCLS recommends use of the microdilution method to determine MICs for pneumococci, our group has successfully performed the agar dilution method for many years, with uniformly satisfactory quality control and other results $(3,10,19,21,22)$. All compounds except daptomycin were tested by the agar dilution method. Daptomycin may not be tested by this method because of problems with calcium regulation in agar (20). The MICs for the 12 strains determined by the time-kill method were confirmed by the standard macrodilution method (17). Standard quality control strains were included in each run (17).

For the time-kill studies, tubes containing $5 \mathrm{ml}$ of cationadjusted Mueller-Hinton broth (BBL Microbiology Systems) with $5 \%$ lysed horse blood and doubling antibiotic concentrations were inoculated with $5 \times 10^{5}$ to $5 \times 10^{6} \mathrm{CFU} / \mathrm{ml}$, and the tubes were incubated at $35^{\circ} \mathrm{C}$ in a shaking water bath. The methods were those described previously $(18,24,25)$, with the addition of calcium for the studies with daptomycin.

The results of the time-kill assays were analyzed by determining the number of strains which yielded changes in the $\log _{10}$ CFU per milliliter of $-1,-2$, and -3 at $0,3,6,12$, and $24 \mathrm{~h}$ compared to the counts at time zero. The lowest concentration of an antimicrobial that reduced the original inoculum by $\geq 3 \log _{10} \mathrm{CFU} / \mathrm{ml}(99.9 \%)$ at each of the time periods was considered bactericidal, and the lowest concentration of an antimicrobial that reduced the original inoculum by 0 to $<3$ $\log _{10} \mathrm{CFU} / \mathrm{ml}$ was considered bacteriostatic. The problem of antibiotic carryover was addressed by dilution, as described previously $(18,24,25)$. Ten strains for which ciprofloxacin MICs were $<8.0 \mu \mathrm{g} / \mathrm{ml}$ were used to test the quinolones by the time-kill method. Only two strains for which erythromycin MICs were $0.03 \mu \mathrm{g} / \mathrm{ml}$ were used in tests with azithromycin and clarithromycin by the time-kill method.

The MICs for the 300 strains classified by their penicillin susceptibilities are summarized in Table 1, and the MICs for 
TABLE 1. Agar dilution MICs for 300 strains classified by penicillin susceptibility

\begin{tabular}{|c|c|c|c|c|c|c|c|}
\hline \multirow{2}{*}{$\begin{array}{l}\text { Drug and penicillin } \\
\text { susceptibility }^{a}\end{array}$} & \multicolumn{3}{|c|}{ MIC ( $\mu \mathrm{g} / \mathrm{ml})$} & \multirow{2}{*}{$\begin{array}{l}\text { Drug and penicillin } \\
\text { susceptibility }\end{array}$} & \multicolumn{3}{|c|}{ MIC $(\mu \mathrm{g} / \mathrm{ml})$} \\
\hline & Range & $50 \%$ & $90 \%$ & & Range & $50 \%$ & 90 \\
\hline Penicillin & & & & Ciprofloxacin & & & \\
\hline $\mathrm{S}$ & $0.016-0.06$ & 0.03 & 0.06 & S & $1.0->32.0$ & 2.0 & 32.0 \\
\hline I & $0.125-1.0$ & 0.25 & 1.0 & I & $0.5-32.0$ & 1.0 & 2.0 \\
\hline $\mathrm{R}$ & $2.0-16.0$ & 2.0 & 4.0 & $\mathrm{R}$ & $0.5->32.0$ & 2.0 & 4.0 \\
\hline LBM415 & & & & Levofloxacin & & & \\
\hline $\mathrm{S}$ & $0.06-4.0$ & 1.0 & 2.0 & S & $1.0-32.0$ & 1.0 & 16.0 \\
\hline I & $0.03-2.0$ & 0.5 & 1.0 & I & $0.5-32.0$ & 1.0 & 2.0 \\
\hline $\mathrm{R}$ & $0.06-4.0$ & 0.5 & 1.0 & $\mathrm{R}$ & $0.5-32.0$ & 1.0 & 2.0 \\
\hline Amoxicillin & & & & Gatifloxacin & & & \\
\hline $\mathrm{S}$ & $\leq 0.016-0.125$ & 0.03 & 0.03 & $\mathrm{~S}$ & $0.25-8.0$ & 0.5 & 4.0 \\
\hline I & $\leq 0.016-2.0$ & 0.25 & 1.0 & I & $0.125-16.0$ & 0.25 & 0.5 \\
\hline $\mathrm{R}$ & $0.25-16.0$ & 2.0 & 8.0 & $\mathrm{R}$ & $0.125-16.0$ & 0.5 & 1.0 \\
\hline Amoxicillin-clavulanate & & & & Moxifloxacin & & & \\
\hline Penicillin S & $\leq 0.016-0.125$ & 0.03 & 0.03 & $\mathrm{~S}$ & $0.125-4.0$ & 0.25 & 4.0 \\
\hline Penicillin I & $0.03-2.0$ & 0.25 & 1.0 & I & $0.06-4.0$ & 0.125 & 0.25 \\
\hline Penicillin R & $0.25-16.0$ & 2.0 & 4.0 & $\mathrm{R}$ & $0.06-4.0$ & 0.25 & 0.5 \\
\hline Imipenem & & & & Azithromycin & & & \\
\hline $\mathrm{S}$ & $\leq 0.004-0.03$ & 0.008 & 0.008 & $\mathrm{~S}$ & $0.03->64.0$ & 2.0 & $>64.0$ \\
\hline I & $0.016-0.25$ & 0.03 & 0.125 & I & $\leq 0.016->64.0$ & 2.0 & $>64.0$ \\
\hline $\mathrm{R}$ & $0.06-2.0$ & 0.25 & 0.25 & $\mathrm{R}$ & $0.06->64.0$ & 0.125 & $>64.0$ \\
\hline Meropenem & & & & Clarithromycin & & & \\
\hline $\mathrm{S}$ & $0.008-0.06$ & 0.016 & 0.016 & $\mathrm{~S}$ & $\leq 0.016->64.0$ & 0.5 & $>64.0$ \\
\hline I & $0.016-0.5$ & 0.06 & 0.25 & I & $\leq 0.016->64.0$ & 1.0 & $>64.0$ \\
\hline $\mathrm{R}$ & $0.125-2.0$ & 0.5 & 0.5 & $\mathrm{R}$ & $\leq 0.016->64.0$ & 0.06 & $>64.0$ \\
\hline Ceftriaxone & & & & Cefpodoxime & & & \\
\hline $\mathrm{S}$ & $0.008-0.25$ & 0.03 & 0.06 & $\mathrm{~S}$ & $0.03-0.5$ & 0.03 & 0.06 \\
\hline I & $0.03-1.0$ & 0.125 & 1.0 & I & $0.06-4.0$ & 0.25 & 2.0 \\
\hline $\mathrm{R}$ & $0.5->8.0$ & 1.0 & 2.0 & $\mathrm{R}$ & 1.0-64.0 & 4.0 & 8.0 \\
\hline Cefuroxime & & & & Cefdinir & & & \\
\hline $\mathrm{S}$ & $0.016-0.5$ & 0.03 & 0.125 & S & $0.016-0.5$ & 0.06 & 0.125 \\
\hline I & $0.125-4.0$ & 0.25 & 4.0 & I & 0.06-8.0 & 0.5 & 4.0 \\
\hline $\mathrm{R}$ & $2.0-64.0$ & 4.0 & 16.0 & $\mathrm{R}$ & $2.0->64.0$ & 8.0 & 8.0 \\
\hline
\end{tabular}

${ }^{a}$ S, susceptible $(n=80)$; I, intermediate $(n=88)$; R, resistant. $(n=132)$.

the 300 strains classified by their macrolide susceptibilities are summarized in Table 2. The LBM415 MICs (range, 0.03 to 4.0 $\mu \mathrm{g} / \mathrm{ml}$; MICs at which $50 \%$ of isolates are inhibited $\left[\mathrm{MIC}_{50} \mathrm{~s}\right.$ ], 0.5 to $1.0 \mu \mathrm{g} / \mathrm{ml} ; \mathrm{MIC}_{90} \mathrm{~s}, 1.0$ to $2.0 \mu \mathrm{g} / \mathrm{ml}$ ) were similar, irrespective of the strain's $\beta$-lactam, macrolide, or quinolone resistance phenotype and genotype. All strains were also susceptible to vancomycin (MICs, 0.03 to $1.0 \mu \mathrm{g} / \mathrm{ml}$ ), teicoplanin (MICs, $\leq 0.016$ to $0.125 \mu \mathrm{g} / \mathrm{ml}$ ), linezolid (MICs, 0.25 to 2.0 $\mu \mathrm{g} / \mathrm{ml}$ ), and quinupristin-dalfopristin (MICs, 0.125 to $1.0 \mu \mathrm{g} /$ $\mathrm{ml}$ ). The MICs of the $\beta$-lactams rose with those of penicillin G. Among the quinolones, moxifloxacin had the lowest MICs for quinolone-susceptible and -resistant strains. The penicillin MICs for macrolide-resistant strains were also generally increased. Because the MICs of the glycopeptides, linezolid, and quinupristin-dalfopristin were similar irrespective of the phenotypic penicillin $\mathrm{G}$ and macrolide resistance data, they are not presented in Tables 1 and 2. The quinolone MICs are listed in 1 to reflect their distributions by penicillin G susceptibility.

The MICs for the strains tested by the time-kill method are listed in Table 3, and the results obtained by the time-kill method are listed in Table 4. LBM415 had killing kinetics similar to those of linezolid; at two times the MIC, it was bactericidal (99.9\% killing) against six strains after $24 \mathrm{~h}$. The $\beta$-lactams, quinolones, and glycopeptides were bactericidal against 12,9 to 10 , and 11 to 12 strains, respectively, at four times the MIC after $24 \mathrm{~h}$. Macrolides showed slower killing. More rapid bactericidal activity, with significant killing at earlier time periods, was seen with daptomycin and quinupristindalfopristin.

LBM415 is a new peptide deformylase inhibitor $(1,2,7,12$, 13) with excellent activity against clinically significant grampositive strains, including multiresistant organisms such as linezolid-resistant staphylococci (MIC range, 0.25 to $2 \mu \mathrm{g} / \mathrm{ml}$ ), Enterococcus faecalis (MIC range, 2 to $4 \mu \mathrm{g} / \mathrm{ml}$ ), and Enterococcus faecium, including strains resistant to quinupristin-dalfopristin (MIC range, 0.5 to $4 \mu \mathrm{g} / \mathrm{ml}$ ) (13). In another recent study of 1,837 recent gram-positive strains, the LBM415 MICs for all strains except 6 enterococci ( $0.3 \%$ of strains overall) were $\leq 4$ $\mu \mathrm{g} / \mathrm{ml}$; the MICs for pneumococci ranged from 0.5 to $2 \mu \mathrm{g} / \mathrm{ml}$ (12). A previously published study testing another related peptide 
TABLE 2. Agar dilution MICs for 300 strains classified by macrolide susceptibility MIC $(\mu \mathrm{g} / \mathrm{ml})$

\begin{tabular}{|c|c|c|c|c|c|c|c|}
\hline \multirow{2}{*}{$\begin{array}{l}\text { Drug, macrolide } \\
\text { susceptibility, }{ }^{a} \text { and resistance } \\
\text { mechanism }\end{array}$} & \multicolumn{3}{|c|}{$\operatorname{MIC}(\mu \mathrm{g} / \mathrm{ml})$} & \multirow{2}{*}{$\begin{array}{l}\text { Drug, macrolide susceptibility, } \\
\text { and resistance mechanism }\end{array}$} & \multicolumn{3}{|c|}{$\operatorname{MIC}(\mu \mathrm{g} / \mathrm{ml})$} \\
\hline & Range & $50 \%$ & $90 \%$ & & Range & $50 \%$ & $90 \%$ \\
\hline Penicillin & & & & Ceftriaxone & & & \\
\hline S & $0.016-4.0$ & 1.0 & 2.0 & S & $0.016-4.0$ & 0.5 & 1.0 \\
\hline $\mathrm{R}$ & $0.016-16.0$ & 0.5 & 4.0 & $\mathrm{R}$ & $0.008->8.0$ & 0.25 & 2.0 \\
\hline erm(B) & $0.016-16.0$ & 1.0 & 4.0 & erm(B) & $0.016->8.0$ & 0.5 & 2.0 \\
\hline$m e f(\mathrm{~A})$ & $0.016-8.0$ & 0.06 & 2.0 & $m e f(\mathrm{~A})$ & $0.016-4.0$ & 0.06 & 1.0 \\
\hline $\operatorname{erm}(\mathrm{A})$ & $0.016-0.03$ & & & $\operatorname{erm}(\mathrm{A})$ & 0.03 & & \\
\hline $\operatorname{erm}(\mathrm{B})+m e f(\mathrm{~A})$ & 0.016 & & & $\operatorname{erm}(\mathrm{B})+m e f(\mathrm{~A})$ & 0.03 & & \\
\hline L4 & $2.0-16.0$ & 4.0 & 8.0 & L4 & $0.5->8.0$ & 2.0 & 4.0 \\
\hline $23 \mathrm{~S}$ rRNA & $0.03-0.25$ & & & 23S rRNA & $0.008-0.125$ & & \\
\hline LBM415 & & & & Cefuroxime & & & \\
\hline $\mathrm{S}$ & $0.03-4.0$ & 0.5 & 1.0 & $\mathrm{~S}$ & $0.016-16.0$ & 4.0 & 8.0 \\
\hline $\mathrm{R}$ & $0.03-4.0$ & 0.5 & 2.0 & $\mathrm{R}$ & $0.016-64.0$ & 0.5 & 8.0 \\
\hline erm (B) & $0.06-4.0$ & 0.5 & 1.0 & $\operatorname{erm}(\mathrm{B})$ & $0.016-64.0$ & 4.0 & 8.0 \\
\hline$m e f(\mathrm{~A})$ & $0.03-4.0$ & 0.25 & 2.0 & $m e f(\mathrm{~A})$ & $0.03-8.0$ & 0.125 & 8.0 \\
\hline $\operatorname{erm}(\mathrm{A})$ & $1.0-2.0$ & & & $\operatorname{erm}(\mathrm{A})$ & 0.03 & & \\
\hline$e r m(\mathrm{~B})+m e f(\mathrm{~A})$ & 1.0 & & & $e r m(\mathrm{~B})+m e f(\mathrm{~A})$ & 0.03 & & \\
\hline L4 & $0.125-4.0$ & 0.5 & 2.0 & L4 & $4.0-64.0$ & 8.0 & 32.0 \\
\hline $23 \mathrm{~S}$ rRNA & $0.25-2.0$ & & & $23 \mathrm{~S}$ rRNA & $0.03-0.5$ & & \\
\hline Amoxicillin & & & & Cefpodoxime & & & \\
\hline $\mathrm{S}$ & $\leq 0.016-4.0$ & 1.0 & 2.0 & $\mathrm{~S}$ & $0.03-8.0$ & 1.0 & 4.0 \\
\hline $\mathrm{R}$ & $\leq 0.016-16.0$ & 0.25 & 8.0 & $\mathrm{R}$ & $0.03-64.0$ & 0.5 & 4.0 \\
\hline erm(B) & $\leq 0.016-16.0$ & 1.0 & 8.0 & $\operatorname{erm}(\mathrm{B})$ & $0.03-64.0$ & 2.0 & 4.0 \\
\hline$m e f(\mathrm{~A})$ & $\leq 0.016-4.0$ & 0.06 & 2.0 & $m e f(\mathrm{~A})$ & $0.03-8.0$ & 0.06 & 4.0 \\
\hline $\operatorname{erm}(\mathrm{A})$ & $\leq 0.016-0.03$ & & & $\operatorname{erm}(\mathrm{A})$ & 0.03 & & \\
\hline $\operatorname{erm}(\mathrm{B})+m e f(\mathrm{~A})$ & 0.03 & & & $\operatorname{erm}(\mathrm{B})+m e f(\mathrm{~A})$ & 0.03 & & \\
\hline L4 & $0.25-16.0$ & 4.0 & 8.0 & $\mathrm{~L} 4$ & $2.0-32.0$ & 4.0 & 32.0 \\
\hline 23S rRNA & 0.03 & & & 23S rRNA & $0.03-0.125$ & & \\
\hline Amoxicillin-clavulanate & & & & Cefdinir & & & \\
\hline $\mathrm{S}$ & $\leq 0.016-4.0$ & 1.0 & 2.0 & $\mathrm{~S}$ & $0.016-16.0$ & 4.0 & 8.0 \\
\hline $\mathrm{R}$ & $\leq 0.016-16.0$ & 0.25 & 4.0 & $\mathrm{R}$ & $0.03->64.0$ & 0.5 & 8.0 \\
\hline erm(B) & $\leq 0.016-16.0$ & 1.0 & 4.0 & erm(B) & $0.03->64.0$ & 4.0 & 8.0 \\
\hline$m e f(\mathrm{~A})$ & $\leq 0.016-4.0$ & 0.06 & 2.0 & $m e f(\mathrm{~A})$ & $0.03-16.0$ & 0.125 & 8.0 \\
\hline $\operatorname{erm}(\mathrm{A})$ & $\leq 0.016-0.03$ & & & $\operatorname{erm}(\mathrm{A})$ & 0.06 & & \\
\hline $\operatorname{erm}(\mathrm{B})+m e f(\mathrm{~A})$ & 0.03 & & & $\operatorname{erm}(\mathrm{B})+m e f(\mathrm{~A})$ & 0.06 & & \\
\hline L4 & $0.25-16.0$ & 4.0 & 8.0 & L4 & $4.0-32.0$ & 8.0 & 16.0 \\
\hline 23S rRNA & 0.03 & & & 23S rRNA & $0.06-0.125$ & & \\
\hline Imipenem & & & & Azithromycin & & & \\
\hline $\mathrm{S}$ & $\leq 0.004-0.5$ & 0.125 & 0.25 & $\mathrm{~S}$ & $\leq 0.016-0.125$ & 0.125 & 0.125 \\
\hline $\mathrm{R}$ & $\leq 0.004-2.0$ & 0.06 & 0.25 & $\mathrm{R}$ & $1.0->64.0$ & $>64.0$ & $>64.0$ \\
\hline erm(B) & $\leq 0.004-2.0$ & 0.125 & 0.25 & $\operatorname{erm}(\mathrm{B})$ & $2.0->64.0$ & $>64.0$ & $>64.0$ \\
\hline$m e f(\mathrm{~A})$ & $\leq 0.004-0.5$ & 0.016 & 0.25 & $m e f(\mathrm{~A})$ & $1.0->64.0$ & 4.0 & 16.0 \\
\hline $\operatorname{erm}(\mathrm{A})$ & $\leq 0.004-0.008$ & & & $\operatorname{erm}(\mathrm{A})$ & $4.0-8.0$ & & \\
\hline $\operatorname{erm}(\mathrm{B})+m e f(\mathrm{~A})$ & 0.008 & & & $\operatorname{erm}(\mathrm{B})+\operatorname{mef}(\mathrm{A})$ & $>64.0$ & & \\
\hline L4 & $0.125-0.25$ & 0.25 & 0.25 & L4 & $>64.0$ & $>64.0$ & $>64.0$ \\
\hline 23S rRNA & $\leq 0.004-0.03$ & & & 23S rRNA & $>64.0$ & & \\
\hline Meropenem & & & & Clarithromycin & & & \\
\hline $\mathrm{S}$ & $0.008-1.0$ & 0.25 & 0.5 & $\mathrm{~S}$ & $\leq 0.016-0.06$ & 0.03 & 0.03 \\
\hline $\mathrm{R}$ & $0.008-2.0$ & 0.125 & 0.5 & $\mathrm{R}$ & $0.5->64.0$ & 64.0 & $>64.0$ \\
\hline erm(B) & $0.008-2.0$ & 0.25 & 0.5 & $\operatorname{erm}(\mathrm{B})$ & $0.5->64.0$ & $>64.0$ & $>64.0$ \\
\hline$m e f(\mathrm{~A})$ & $0.016-2.0$ & 0.03 & 0.5 & $m e f(\mathrm{~A})$ & $0.5-32.0$ & 2.0 & 8.0 \\
\hline $\operatorname{erm}(\mathrm{A})$ & $0.008-0.016$ & & & $\operatorname{erm}(\mathrm{A})$ & $0.5-1.0$ & & \\
\hline $\operatorname{erm}(\mathrm{B})+m e f(\mathrm{~A})$ & 0.016 & & & $\operatorname{erm}(\mathrm{B})+m e f(\mathrm{~A})$ & $>64.0$ & & \\
\hline $\mathrm{L} 4$ & $0.125-1.0$ & 0.5 & 0.5 & $\mathrm{~L} 4$ & $8.0-64.0$ & 32.0 & 64.0 \\
\hline $23 \mathrm{~S}$ rRNA & $0.016-0.06$ & & & 23S rRNA & $8.0-16.0$ & & \\
\hline
\end{tabular}

${ }^{a} \mathrm{~S}$, susceptible $(n=146)$; , resistant $(n=154)$. The 154 resistant isolates had the following resistance mechanisms: $\operatorname{erm}(\mathrm{B}), n=87 ; m e f(\mathrm{~A}), n=40 ; \operatorname{erm}(\mathrm{A}), n$ $=4 ; \operatorname{erm}(\mathrm{B})$ and $m e f(\mathrm{~A}), n=1 ; \mathrm{L} 4$ protein mutation (L4), $n=18$; and 23S rRNA mutation (23S rRNA), $n=4$. 
TABLE 3. MICs for 12 strains tested by time-kill method

\begin{tabular}{|c|c|c|c|c|c|c|c|c|c|c|c|c|}
\hline \multirow{2}{*}{ Drug } & \multicolumn{12}{|c|}{ MIC $(\mu \mathrm{g} / \mathrm{ml})$ for strain: } \\
\hline & $1^{a}$ & $2^{a}$ & $3^{a}$ & $4^{a}$ & $5^{b}$ & $6^{b}$ & $7^{b}$ & $8^{b}$ & $9^{c}$ & $10^{c}$ & $11^{d}$ & $12^{d}$ \\
\hline LBM415 & 0.12 & 2.0 & 2.0 & 2.0 & 0.12 & 0.25 & 0.5 & 0.25 & 0.5 & 0.5 & 0.5 & 1.0 \\
\hline Amoxicillin & 0.016 & 2.0 & 2.0 & 2.0 & 0.25 & 0.03 & 0.06 & 0.06 & 2.0 & 2.0 & 2.0 & 0.03 \\
\hline Amoxicillin-clavulanate & 0.016 & 2.0 & 2.0 & 2.0 & 0.12 & 0.03 & 0.03 & 0.12 & 2.0 & 2.0 & 2.0 & 0.016 \\
\hline Imipenem & 0.004 & 0.25 & 0.25 & 0.25 & 0.03 & 0.008 & 0.008 & 0.03 & 0.25 & 0.12 & 0.25 & 0.008 \\
\hline Meropenem & 0.008 & 0.5 & 0.5 & 1.0 & 0.06 & 0.016 & 0.016 & 0.06 & 0.5 & 0.25 & 0.5 & 0.016 \\
\hline Ceftriaxone & 0.016 & 2.0 & 2.0 & 2.0 & 0.12 & 0.016 & 0.06 & 0.06 & 2.0 & 2.0 & 4.0 & 0.03 \\
\hline Cefuroxime & 0.016 & 2.0 & 2.0 & 4.0 & 0.25 & 0.03 & 0.12 & 0.25 & 8.0 & 16.0 & 16.0 & 0.03 \\
\hline Cefdinir & 0.06 & 4.0 & 4.0 & 4.0 & 0.12 & 0.12 & 0.03 & 0.06 & 8.0 & 8.0 & 16.0 & 0.12 \\
\hline Ciprofloxacin & 1.0 & 1.0 & 1.0 & 1.0 & 1.0 & 4.0 & 4.0 & 2.0 & 2.0 & 1.0 & 8.0 & $>32.0$ \\
\hline Levofloxacin & 1.0 & 1.0 & 1.0 & 1.0 & 1.0 & 2.0 & 1.0 & 1.0 & 2.0 & 1.0 & 8.0 & 16.0 \\
\hline Gatifloxacin & 1.0 & 0.5 & 0.5 & 0.5 & 0.5 & 0.5 & 0.5 & 0.25 & 0.5 & 0.5 & 4.0 & 8.0 \\
\hline Moxifloxacin & 0.12 & 0.25 & 0.25 & 0.25 & 0.25 & 0.25 & 0.25 & 0.12 & 0.25 & 0.25 & 2.0 & 4.0 \\
\hline Azithromycin & $>64$ & $>64$ & $>64$ & $>64$ & 8.0 & 8.0 & 16.0 & 2.0 & $>64$ & $>64$ & 0.06 & 0.125 \\
\hline Clarithromycin & 64 & $>64$ & $>64$ & $>64$ & 2.0 & 4.0 & 8.0 & 2.0 & 16 & 32 & 0.016 & 0.03 \\
\hline Linezolid & 0.5 & 2.0 & 1.0 & 1.0 & 0.5 & 1.0 & 1.0 & 0.5 & 2.0 & 2.0 & 1.0 & 1.0 \\
\hline Quinupristin-dalfopristin & 0.5 & 1.0 & 0.5 & 0.5 & 0.25 & 0.25 & 0.5 & 0.25 & 0.5 & 0.5 & 0.25 & 0.25 \\
\hline Daptomycin & 0.12 & 0.25 & 0.25 & 0.5 & 0.12 & 0.25 & 0.25 & 0.25 & 0.25 & 0.25 & 0.12 & 0.25 \\
\hline Vancomycin & 0.25 & 0.5 & 0.5 & 0.25 & 0.25 & 0.25 & 0.25 & 0.25 & 0.25 & 0.25 & 0.25 & 0.5 \\
\hline Teicoplanin & 0.06 & 0.12 & 0.06 & 0.12 & 0.06 & 0.12 & 0.12 & 0.06 & 0.06 & 0.12 & 0.12 & 0.06 \\
\hline
\end{tabular}

${ }^{a}$ Macrolide-resistant strains carrying the erm(B) mutation.

${ }^{b}$ Macrolide-resistant strains carrying the $\operatorname{mef}(\mathrm{A})$ mutation.

${ }^{c}$ Macrolide-resistant strains carrying an L4 protein mutation.

${ }^{d}$ Quinolone-resistant strains (ciprofloxacin MIC, $\geq 8.0 \mu \mathrm{g} / \mathrm{ml}$ ).

TABLE 4. Pneumococcal killing kinetics results

\begin{tabular}{|c|c|c|c|c|c|c|c|c|c|c|c|c|}
\hline \multirow{3}{*}{ Drug and multiple of MIC } & \multicolumn{12}{|c|}{ No. of isolates for which the level of killing ${ }^{a}$ was as indicated at the following times: } \\
\hline & \multicolumn{3}{|c|}{$3 \mathrm{~h}$} & \multicolumn{3}{|c|}{$6 \mathrm{~h}$} & \multicolumn{3}{|c|}{$12 \mathrm{~h}$} & \multicolumn{3}{|c|}{$24 \mathrm{~h}$} \\
\hline & -1 & -2 & -3 & -1 & -2 & -3 & -1 & -2 & -3 & -1 & -2 & -3 \\
\hline \multicolumn{13}{|l|}{ LBM415 } \\
\hline 4 & 0 & 0 & 0 & 4 & 1 & 0 & 9 & 2 & 2 & 12 & 9 & 8 \\
\hline 2 & 0 & 0 & 0 & 3 & 1 & 0 & 9 & 2 & 2 & 10 & 8 & 6 \\
\hline 1 & 0 & 0 & 0 & 2 & 0 & 0 & 8 & 1 & 1 & 7 & 4 & 2 \\
\hline \multicolumn{13}{|l|}{ Amoxicillin } \\
\hline 4 & 12 & 7 & 0 & 12 & 11 & 6 & 12 & 12 & 11 & 12 & 12 & 12 \\
\hline 2 & 9 & 4 & 0 & 12 & 10 & 6 & 12 & 11 & 10 & 12 & 12 & 12 \\
\hline 1 & 7 & 3 & 0 & 10 & 9 & 3 & 12 & 10 & 8 & 12 & 11 & 9 \\
\hline \multicolumn{13}{|l|}{ Amoxicillin-clavulanate } \\
\hline 4 & 11 & 6 & 1 & 12 & 11 & 7 & 12 & 11 & 10 & 12 & 12 & 12 \\
\hline 2 & 10 & 6 & 1 & 12 & 11 & 7 & 12 & 11 & 10 & 12 & 12 & 12 \\
\hline 1 & 9 & 3 & 1 & 12 & 10 & 3 & 12 & 11 & 9 & 11 & 11 & 10 \\
\hline \multicolumn{13}{|l|}{ Imipenem } \\
\hline 4 & 12 & 7 & 0 & 12 & 10 & 5 & 12 & 12 & 11 & 12 & 12 & 12 \\
\hline 2 & 12 & 5 & 0 & 12 & 10 & 5 & 12 & 12 & 11 & 12 & 12 & 12 \\
\hline 1 & 11 & 4 & 0 & 11 & 6 & 4 & 12 & 11 & 8 & 12 & 11 & 10 \\
\hline \multicolumn{13}{|l|}{ Meropenem } \\
\hline 4 & 11 & 4 & 2 & 12 & 9 & 3 & 12 & 12 & 10 & 12 & 12 & 12 \\
\hline 2 & 9 & 3 & 1 & 12 & 7 & 3 & 12 & 11 & 9 & 12 & 12 & 12 \\
\hline 1 & 8 & 2 & 1 & 11 & 7 & 3 & 11 & 7 & 4 & 8 & 7 & 5 \\
\hline \multicolumn{13}{|l|}{ Ceftriaxone } \\
\hline 4 & 7 & 2 & 0 & 12 & 6 & 2 & 12 & 12 & 10 & 12 & 12 & 12 \\
\hline 2 & 6 & 2 & 0 & 12 & 7 & 3 & 12 & 12 & 10 & 12 & 11 & 10 \\
\hline 1 & 4 & 1 & 0 & 8 & 5 & 3 & 9 & 9 & 6 & 11 & 8 & 4 \\
\hline \multicolumn{13}{|l|}{ Cefuroxime } \\
\hline 4 & 11 & 3 & 2 & 12 & 10 & 3 & 12 & 11 & 10 & 12 & 12 & 12 \\
\hline 2 & 11 & 3 & 0 & 12 & 9 & 3 & 12 & 11 & 10 & 12 & 12 & 11 \\
\hline 1 & 9 & 1 & 0 & 12 & 9 & 2 & 12 & 11 & 9 & 11 & 10 & 9 \\
\hline
\end{tabular}


TABLE 4-Continued

\begin{tabular}{|c|c|c|c|c|c|c|c|c|c|c|c|c|}
\hline \multirow{3}{*}{ Drug and multiple of MIC } & \multicolumn{12}{|c|}{ No. of isolates for which the level of killing ${ }^{a}$ was as indicated at the following times: } \\
\hline & \multicolumn{3}{|c|}{$3 \mathrm{~h}$} & \multicolumn{3}{|c|}{$6 \mathrm{~h}$} & \multicolumn{3}{|c|}{$12 \mathrm{~h}$} & \multicolumn{3}{|c|}{$24 \mathrm{~h}$} \\
\hline & -1 & -2 & -3 & -1 & -2 & -3 & -1 & -2 & -3 & -1 & -2 & -3 \\
\hline \multicolumn{13}{|l|}{ Cepodoxime } \\
\hline 4 & 11 & 3 & 0 & 12 & 12 & 4 & 12 & 12 & 10 & 12 & 12 & 12 \\
\hline 2 & 11 & 3 & 0 & 12 & 12 & 3 & 12 & 12 & 10 & 12 & 12 & 12 \\
\hline 1 & 9 & 2 & 0 & 12 & 10 & 3 & 12 & 10 & 9 & 12 & 11 & 11 \\
\hline \multicolumn{13}{|l|}{ Cefdinir } \\
\hline 4 & 10 & 5 & 1 & 12 & 9 & 5 & 12 & 12 & 11 & 12 & 12 & 12 \\
\hline 2 & 9 & 3 & 0 & 12 & 9 & 5 & 12 & 11 & 10 & 12 & 12 & 12 \\
\hline 1 & 4 & 3 & 0 & 10 & 6 & 2 & 12 & 7 & 4 & 9 & 7 & \\
\hline
\end{tabular}

Ciprofloxacin $^{b}$

\begin{tabular}{|c|c|c|c|c|c|c|c|c|c|c|c|c|}
\hline 4 & 9 & 6 & 0 & 10 & 10 & 4 & 10 & 10 & 10 & 10 & 10 & 10 \\
\hline 2 & 9 & 3 & 0 & 10 & 10 & 3 & 10 & 10 & 7 & 10 & 9 & 9 \\
\hline 1 & 6 & 1 & 0 & 9 & 6 & 1 & 10 & 9 & 4 & 8 & 7 & 5 \\
\hline
\end{tabular}

Levofloxacin $^{b}$

$$
\begin{aligned}
& 4 \\
& 2 \\
& 1
\end{aligned}
$$

$\begin{array}{llll}7 & 2 & 0 & 10 \\ 5 & 0 & 0 & 10 \\ 5 & 0 & 0 & 8\end{array}$

\begin{tabular}{|c|c|c|c|c|c|c|}
\hline 10 & 3 & 10 & 10 & 10 & 10 & 10 \\
\hline 10 & 1 & 10 & 10 & 6 & 10 & 10 \\
\hline 4 & 1 & 10 & 8 & 3 & 9 & 9 \\
\hline
\end{tabular}

Gatifloxacin $^{b}$

$$
\begin{aligned}
& 4 \\
& 2 \\
& 1
\end{aligned}
$$

$\begin{array}{rl}10 & 3 \\ 6 & 1 \\ 4 & 0\end{array}$

0
0
0

$\begin{array}{rrrr}10 & 8 & 1 & 10 \\ 10 & 7 & 1 & 10 \\ 7 & 2 & 1 & 10\end{array}$

$\begin{array}{rrrrrr}10 & 10 & 8 & 10 & 10 & 9 \\ 10 & 10 & 7 & 10 & 10 & 9 \\ 10 & 6 & 3 & 9 & 8 & 4\end{array}$

Moxifloxacin $^{b}$

Moxifloxacin

2
1

Azithromycin ${ }^{c}$

$$
\begin{aligned}
& 4 \\
& 2 \\
& 1
\end{aligned}
$$

Clarithromycin ${ }^{c}$

$$
\begin{aligned}
& 4 \\
& 2 \\
& 1
\end{aligned}
$$

Linezolid

$$
\begin{aligned}
& 4 \\
& 2 \\
& 1
\end{aligned}
$$

Quinupristin-

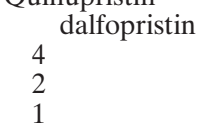
Daptomycin

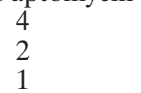

Vancomycin

\begin{tabular}{|c|c|c|c|c|c|}
\hline 10 & 10 & 9 & 10 & 10 & 10 \\
\hline 10 & 10 & 9 & 10 & 10 & 10 \\
\hline 9 & 8 & 5 & 10 & 8 & 7 \\
\hline
\end{tabular}<smiles>[Te][Te]</smiles>

\section{Teicoplanin}

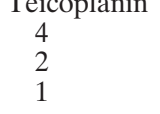

$\begin{array}{rll}10 & 3 & 0 \\ 8 & 1 & 0 \\ 5 & 0 & 0\end{array}$

$\begin{array}{rrrr}10 & 10 & 3 & 10 \\ 10 & 6 & 2 & 10 \\ 8 & 4 & 1\end{array}$

\begin{tabular}{|c|c|c|c|c|c|c|c|}
\hline 11 & 9 & 12 & 11 & 11 & 12 & 12 & 12 \\
\hline 11 & 9 & 12 & 11 & 11 & 12 & 12 & 12 \\
\hline 11 & 6 & 11 & 10 & 7 & 7 & 5 & 3 \\
\hline
\end{tabular}

\begin{tabular}{|c|c|c|c|c|c|c|c|c|c|c|c|}
\hline 11 & 8 & 2 & 12 & 11 & 9 & 12 & 11 & 11 & 12 & 12 & 12 \\
\hline 10 & 4 & 2 & 12 & 11 & 8 & 12 & 11 & 11 & 12 & 11 & 11 \\
\hline 7 & 2 & 2 & 12 & 11 & 4 & 11 & 10 & 7 & 8 & 5 & 3 \\
\hline
\end{tabular}

$\begin{array}{lllllllll}2 & 1 & 1 & 2 & 2 & 2 & 2 & 2 & 2 \\ 1 & 1 & 1 & 2 & 2 & 2 & 2 & 2 & 2 \\ 1 & 1 & 1 & 2 & 1 & 1 & 2 & 2 & 2\end{array}$

\begin{tabular}{|c|c|c|c|c|c|c|c|c|c|c|c|}
\hline 4 & 2 & 0 & 12 & 6 & 3 & 12 & 11 & 10 & 12 & 12 & 12 \\
\hline 4 & 1 & 0 & 12 & 6 & 3 & 12 & 11 & 9 & 12 & 12 & 12 \\
\hline 4 & 0 & 0 & 12 & 5 & 2 & 12 & 11 & 9 & 11 & 10 & 9 \\
\hline
\end{tabular}

$\begin{array}{llllllllllll}0 & 0 & 0 & 2 & 2 & 0 & 2 & 2 & 2 & 2 & 2 & 2 \\ 0 & 0 & 0 & 2 & 2 & 0 & 2 & 2 & 2 & 2 & 2 & 2 \\ 0 & 0 & 0 & 2 & 1 & 0 & 2 & 2 & 2 & 2 & 2 & 2\end{array}$

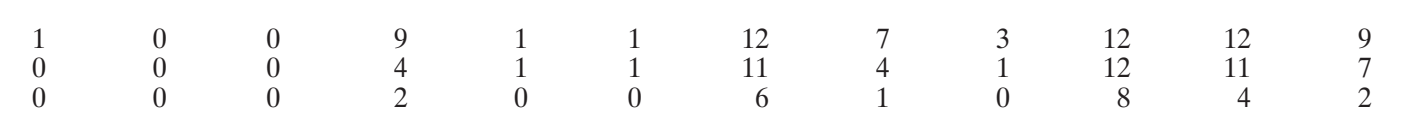

$\begin{array}{rrrr}11 & 10 & 6 & 12 \\ 11 & 9 & 5 & 12 \\ 10 & 7 & 4 & 12\end{array}$

${ }^{a}-1,-1 \log _{10} \mathrm{CFU} / \mathrm{ml}\left(90 \%\right.$ killing); $-2,-2 \log _{10} \mathrm{CFU} / \mathrm{ml}$ (99\% killing); $-3,-3 \log _{10} \mathrm{CFU} / \mathrm{ml}$ (99.9\% killing).
${ }^{b}$ Ten strains for which the ciprofloxacin MIC was $<8.0 \mu \mathrm{g} / \mathrm{ml}$ were tested.

${ }^{c}$ Two macrolide-sensitive strains (erythromycin MIC, $0.03 \mu \mathrm{g} / \mathrm{ml}$ ) were tested. 
deformylase inhibitor, NVP-PDF386 (VCR4887), showed MICs for $\beta$-lactam- and macrolide-susceptible and -resistant pneumococci which ranged from $\leq 0.016$ to $1.0 \mu \mathrm{g} / \mathrm{ml}$, with an $\mathrm{MIC}_{50}$ of $0.25 \mu \mathrm{g} / \mathrm{ml}$ and an $\mathrm{MIC}_{90}$ of $0.5 \mu \mathrm{g} / \mathrm{ml}$ (14). As was the case with our study, no differences in NVP-PDF386 MICs were found between drug-resistant and drug-susceptible pneumococcal strains (14). The results of the present study confirm the low MICs of LBM415 for pneumococci. Susceptibility breakpoints have not yet been established for LBM415, so the significance of the MIC range cannot be interpreted at this time. However, all strains were susceptible (MICs, $\leq 4.0 \mu \mathrm{g} / \mathrm{ml}$ ).

LBM415 was slowly bactericidal, with $99.9 \%$ killing of 8 of 12 strains at four times the MIC after $24 \mathrm{~h}$, with slower rates of killing at lower concentrations and earlier time periods. By comparison, daptomycin and quinupristin-dalfopristin have the most rapid killing, followed by the quinolones, the $\beta$-lactams, and the macrolides. The results for the last three groups of compounds have been published previously $(4,5,8-11,16$, 18-25).

Craig and Andes (W. A. Craig and D. R. Andes, Abstr. 14th Eur. Congr. Clin. Microbiol. Infect. Dis., abstr. P921 and P922, 2004) have shown that the $24-\mathrm{h}$ area under the concentrationtime curve (AUC)/MIC ratio is the parameter that best correlates with the in vivo activity of LBM415 in mice. The drug also has a prolonged in vivo postantibiotic effect, suggesting twicedaily dosing. For pneumococci, the mean free AUC/MIC ratio was $34.9 \pm 7.1$, with bacteriostatic doses ranging from 47.6 to $81.3 \mathrm{mg} / \mathrm{kg}$ of body weight $/ 6 \mathrm{~h}$.

The results of this study indicate a potential role for LBM415 in the treatment of pneumococcal infections. However, interpretation of these in vitro results must be complemented by toxicity and human pharmacokinetic and pharmacodynamic studies (not available at present) before the drug can be recommended for clinical testing.

This study was supported by a grant from Novartis Research Laboratories.

\section{REFERENCES}

1. Anderegg, T. R., D. J. Biedenbach, R. N. Jones, and the Quality Control Working Group. 2003. Quality control guidelines for MIC susceptibility testing of NVP-PDF713, a novel peptide deformylase inhibitor. Int. J. Antimicrob. Agents 22:84-86.

2. Anderegg, T. R., R. N. Jones, and the Quality Control Working Group. 2004. Disk diffusion quality control guidelines for NVP-PDF 713: a novel peptide deformylase inhibitor. Diagn. Microbiol. Infect. Dis. 48:55-57.

3. Appelbaum, P. C. 1992. Antimicrobial resistance in Streptococcus pneumoniae - an overview. Clin. Infect. Dis. 15:77-83.

4. Cercenado, E., F. Garcia-Garrote, and E. Bouza. 2001. In-vitro activity of linezolid against multiply resistant gram-positive clinical isolates. J. Antimicrob. Chemother. 47:77-81.

5. Diekema, D. J., and R. N. Jones. 2000. Oxazolidinones. A review. Drugs 59:7-16.

6. Friedland, I. R., and G. H. McCracken, Jr. 1994. Management of infections caused by antibiotic-resistant Streptococcus pneumoniae. N. Engl. J. Med. 331:377-382.

7. Fritsche, T. R., G. J. Moet, and R. N. Jones. Commercial broth microdilution panel validation and reproducibility trials for NVP-PDF 713, a novel inhibitor of bacterial peptide deformylase. Clin. Microbiol. Infect., in press.

8. Henwood, C. J., D. M. Livermore, A. P. Johnson, D. James, M. Warner, A. Gardiner, and the Linezolid Study Group. 2000. Susceptibility of grampositive cocci from 25 UK hospitals to antimicrobial agents including linezolid. J. Antimicrob. Chemother. 46:931-940.

9. Hoellman, D. B., G. Lin, L. M. Ednie, A. Rattan, M. R. Jacobs, and P. C. Appelbaum. 2003. Antipneumococcal and antistaphylococcal activities of ranbezolid (RBX 7644), a new oxazolidinone, compared to those of other agents. Antimicrob. Agents Chemother. 47:1148-1150.

10. Jacobs, M. R., and P. C. Appelbaum. 1995. Antibiotic-resistant pneumococci. Rev. Med. Microbiol. 6:77-93.

11. Jacobs, M. R., S. Bajaksouzian, A. Zilles, G. Lin, G. A. Pankuch, and P. C. Appelbaum. 1999. Susceptibilities of Streptococcus pneumoniae and Haemophilus influenzae to 10 oral antimicrobial agents based on pharmacodynamic parameters: 1997 U.S. surveillance study. Antimicrob. Agents Chemother. 43:1901-1908.

12. Jones, R. N., T. R. Fritsche, and H. S. Sader. 2004. Antimicrobial spectrum and activity of NVP-PDF 713, a novel peptide deformylase inhibitor, tested against 1,837 recent gram-positive clinical isolates. Diagn. Microbiol. Infect. Dis. 49:63-65.

13. Jones, R. N., G. J. Moet, H. S. Sader, and T. R. Fritsche. 2004. Potential utility of a peptide deformylase inhibitor (NVP-PDF 713) against oxazolidinone-resistant or streptogramin-resistant gram-positive organism isolates. J. Antimicrob. Chemother. 53:804-807.

14. Jones, R. N., and P. R. Rhomberg. 2003. Comparative spectrum and activity of NVP-PDF386 (VRC4887), a new peptide deformylase inhibitor. J. Antimicrob. Chemother. 51:157-161.

15. Liñares, J., A. G. Campa, and R. Pallares. 1999. Fluoroquinolone-resistance in Streptococcus pneumoniae. N. Engl. J. Med. 20:1546-1548.

16. Livermore, D. M. 2000. Quinupristin/dalfopristin and linezolid: where, when, which and whether to use? J. Antimicrob. Chemother. 46:347-350.

17. National Committee for Clinical Laboratory Standards. 2003. Methods for dilution antimicrobial susceptibility tests for bacteria that grow aerobically, 6th ed.; approved standard. NCCLS publication no. M7-A6. National Committee for Clinical Laboratory Standards, Wayne, Pa.

18. Pankuch, G. A., M. R. Jacobs, and P. C. Appelbaum. 1994. Study of comparative antipneumococcal activities of penicillin G, RP 59500, erythromycin, sparfloxacin, ciprofloxacin, and vancomycin by using time-kill methodology. Antimicrob. Agents Chemother. 38:2065-2072.

19. Pankuch, G. A., M. R. Jacobs, and P. C. Appelbaum. 1995. Comparative activity of ampicillin, amoxycillin, amoxycillin/clavulanate and cefotaxime against 189 penicillin-susceptible and -resistant pneumococci. J. Antimicrob. Chemother. 35:883-888.

20. Pankuch, G. A., M. R. Jacobs, and P. C. Appelbaum. 2003. Bactericidal activity of daptomycin against Streptococcus pneumoniae compared with eight other antimicrobials. J. Antimicrob. Chemother. 51:443-446.

21. Spangler, S. K., M. R. Jacobs, and P. C. Appelbaum. 1992. Susceptibilities of penicillin-susceptible and -resistant strains of Streptococcus pneumoniae to RP 59500, vancomycin, erythromycin, PD 131628, sparfloxacin, temafloxacin, Win 57273, ofloxacin, and ciprofloxacin. Antimicrob. Agents Chemother. 36:856-859.

22. Spangler, S. K., M. R. Jacobs, G. A. Pankuch, and P. C. Appelbaum. 1993. Susceptibility of 170 penicillin-susceptible and -resistant pneumococci to six oral cephalosporins, four quinolones, desacetylcefotaxime, Ro 23-9424 and RP 67829. J. Antimicrob. Chemother. 31:273-280.

23. Stevens, D. L., L. G. Smith, J. B. Bruss, M. A. McConnell-Martin, S. E. Duvall, W. M. Todd, and B. Hafkin. 2000. Randomized comparison of linezolid (PNU-100766) versus oxacillin-dicloxacillin for treatment of complicated skin and soft tissue infections. Antimicrob. Agents Chemother. 44:3408-3413.

24. Visalli, M. A., M. R. Jacobs, and P. C. Appelbaum. 1996. MIC and time-kill study of DU-6859a, ciprofloxacin, levofloxacin, sparfloxacin, cefotaxime, imipenem, and vancomycin against nine penicillin-susceptible and -resistant pneumococci. Antimicrob. Agents Chemother. 40:362366 .

25. Visalli, M. A., M. R. Jacobs, and P. C. Appelbaum. 1997. Antipneumococcal activity of BAY 12-8039, a new quinolone, compared with activities of three other quinolones and four $\beta$-lactams. Antimicrob. Agents Chemother. 41: 2786-2789. 